\title{
Becoming overweight: is there a health risk?
}

\author{
Ginter $E^{1}$, Simko $\mathrm{V}^{2}$ \\ Slovak Medical University, Bratislava, Slovakia. ginter.emil@mail.t-com.sk
}

\begin{abstract}
Overweight and obesity is becoming widespread enough to generate an acceptable and misleading social status. By 2030, in the USA up to $86 \%$ of adults will be overweight or obese. Some selected statistical data based on the body mass index (BMI) indicated that overweight was not associated with increased mortality, this provoked a conceivable interest. Added to this is the observation that while the prevalence of obesity is dramatically increasing, the cardiovascular mortality and life expectancy in the European Union and USA has improved. When more sensitive indicators of body adiposity and its distribution than the BMI, like the waist-thigh ratio in both sexes and the waist-hip ratio in women are projected on mortality, it becomes obvious that even overweight is associated with an increased health risk. Gaining excessive body fat is a continuous, frequently progressive process. Present obesity epidemic in childhood will manifest with deleterious consequences only in future years when adolescents reach adulthood. Prevention is thus essential even before the overweight sets in. Improved life expectancy observed in large populations despite obesity epidemic, is a favourable medical success in the management of hypertension, of serum lipid disorders and diabetes. While encouraging, when it is observed in large population, it does not take away the potential health risk of a metabolic disorder in an individual who is overweight (Fig. 7, Ref. 25). Text in PDF www.elis.sk.

Key words: overweight, obesity, body mass index, diabetes type 2, hypertension, cardiovascular disorders, life expectancy.
\end{abstract}

\section{Globalization of overweight and obesity}

Globalization benefits the world with more readily available food supply. At the same time, it promotes overweight and obesity by indiscriminate overuse of food energy. Pandemic of obesity and related metabolic consequences, especially diabetes type 2 , is rapidly becoming a global health problem (1). Developing countries are undergoing rapid nutrition transition, along with increases in overweight, obesity and in the metabolic disorders (2). In the USA, obesity prevalence has increased at an alarming rate, from $13 \%$ to $32 \%$ between the 1960 s and year 2004 (3). Currently, $66 \%$ of US adults are overweight or obese, $16 \%$ of children and adolescents are overweight. Projections of future prevalence of obesity and related health care costs indicate that if present trends continue, by 2030 in the USA up to $86 \%$ adults will be overweight or obese and $51 \%$ will be severely obese (4).

What is the health risk of being overweight or gaining body mass to be manifestly obese? There are multiple reports that unequivocally associate overweight and obesity with impaired health, related to hypertension, cardiovascular disease and diabetes type 2. A total of 33 US studies were reviewed and revealed the direct medical cost of overweight and obesity (5). In 2008, the per-person direct medical cost for overweight was \$ 266 and for obesity it was $\$ 1723$. The aggregate US national cost of overweight and obe-

${ }^{1}$ Slovak Medical University, Bratislava, Slovakia, and ${ }^{2}$ State University New York, Downstate Medical Center at Brooklyn, USA

Address for correspondence: E. Ginter, RND, DSc, Racianska 17, SK83102 Bratislava, Slovakia sity was $\$ 113.9$ billion. The economic burden of morbid obesity (BMI $>$ or $=40 \mathrm{~kg} /$ square meter) is even substantially higher, due to increased morbidity and mortality. Obesity is associated with poor health-related quality of life (6).

\section{Epidemic of overweight and obesity while life expectancy is improving: paradox or reality?}

Closer review of trends associating overweight and obesity with mortality in the USA provides a seemingly challenging paradox: despite all predictions of negative impact of overweight and obesity on health, the life expectancy (LE) in the USA has been improving along with the increasing body weight of the population (Fig. 1). Similar increase of LE was observed in European countries with a high prevalence of overweight and obesity, e.g. in the Czech Republic, England, Germany and Austria. We took note of this discrepancy in our previous report on paradoxes in contemporary medicine (9).

Mortality and LE are essential statistical indicators of population health. The markers of obesity correlated with mortality are either the body mass index (BMI, body weight in kg/body height in square meter) or direct anthropometric measures of body adiposity and fat distribution, the waist-hip ratio (WHR) in women and the waist- thigh ratio (WTR) in both sexes. Using the BMI is a generally accepted measure of categories of body mass. Another measure, WHR has also been used as an indicator or measure of the health of a person and of the risk of developing serious health conditions. Research suggested that people with "apple-shaped" bodies (with more weight around the waist) face more health risks 
Trends of overweight and obesity

(males + females) in the USA

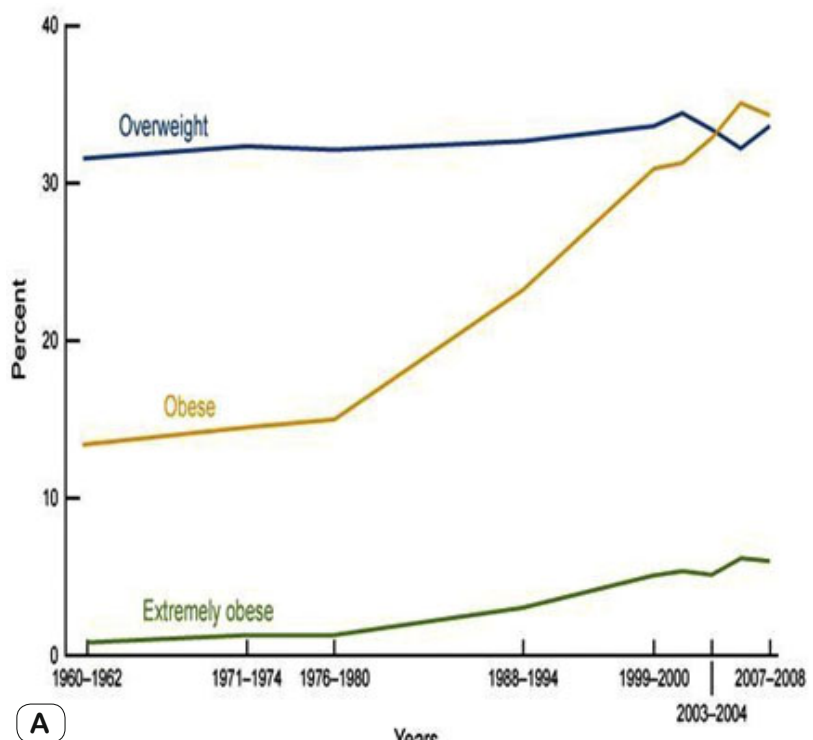

Permanent increase of life expectancy in USA

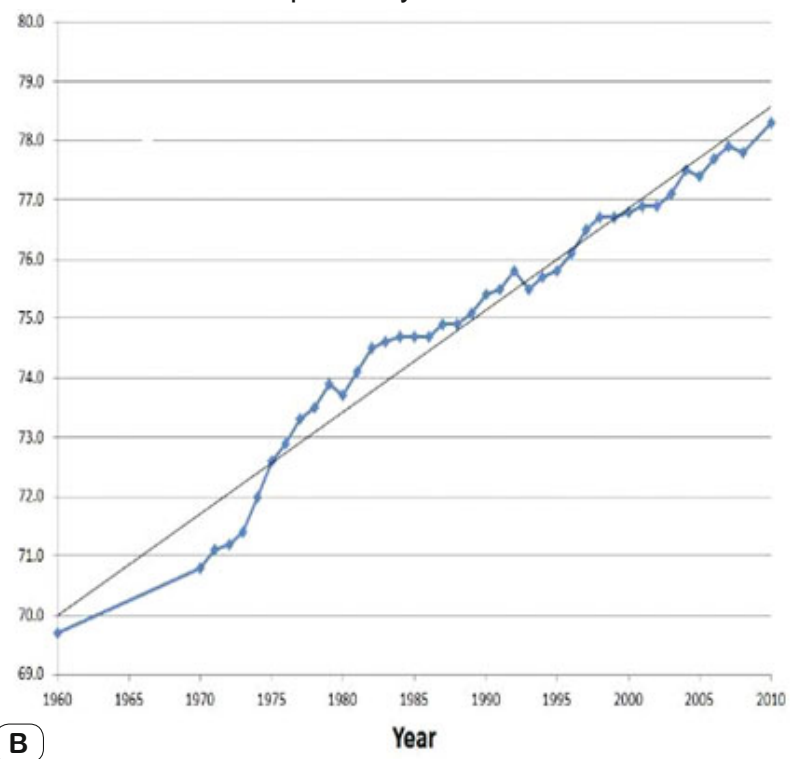

Fig. 1 A-B. PARADOX: Parallel increase of obesity prevalence and Life expectancy in USA. Trends of overweight and obesity along with progressive improvement of life expectancy in the USA. According to National Center for Health Statistics (7) and Statistical Abstract of the United States: 2012 (8).

than those with "pear-shaped" bodies who carry more weight around the hips.

The large study (10) reported a relation of BMI to relative risk of mortality in 1.46 million US whites (Fig. 2). Typical J-shaped relation reflected a higher mortality in population with abnormally low body weight, then lowest mortality at normal weight, mild increase at overweight and a rapid rise of mortality in obese. Significantly increased mortality was only associated with either extreme of BMI.

Different association between BMI and relative mortality risk was found in the very large meta-analysis in 2013 (11). The researchers analyzed 97 published studies of heterogenous populations. 41 were from the United States or Canada and 37 were from Europe. Others were from Australia, China, Japan, Brazil, Israel,

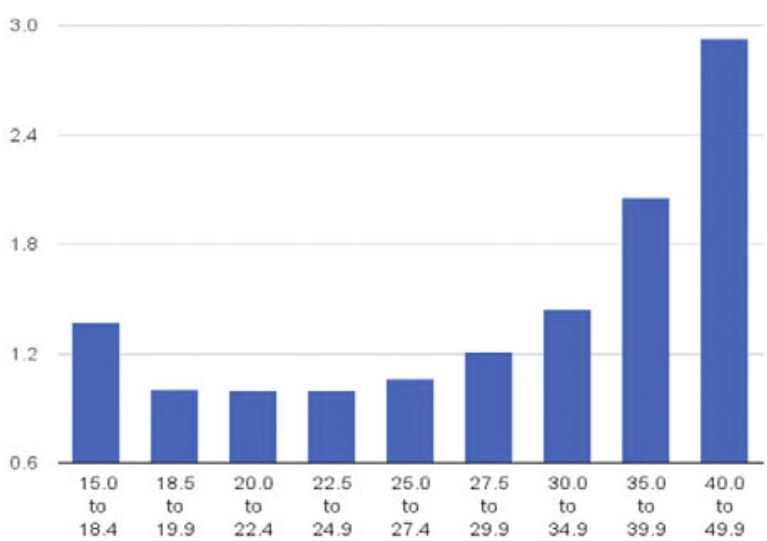

Fig. 2. Relative risk of mortality by BMI in white US men is J-shaped. According to Berrington de Gonzalez et al (10)

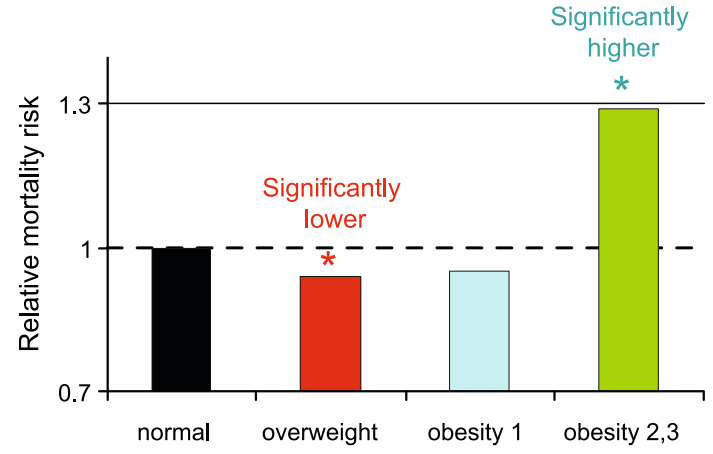

Fig. 3. Relative total mortality risk associated with normal weight, overweight and obesity. According to Flegal et al (11).

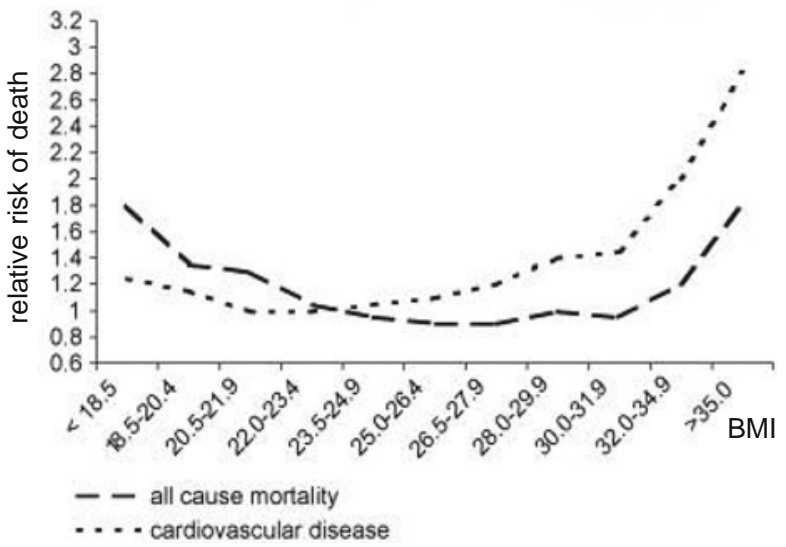

Fig. 4. Paradox of different trends in all-cause and cardiovascular mortality related to increase in BMI. According to Knecht et al (20). 


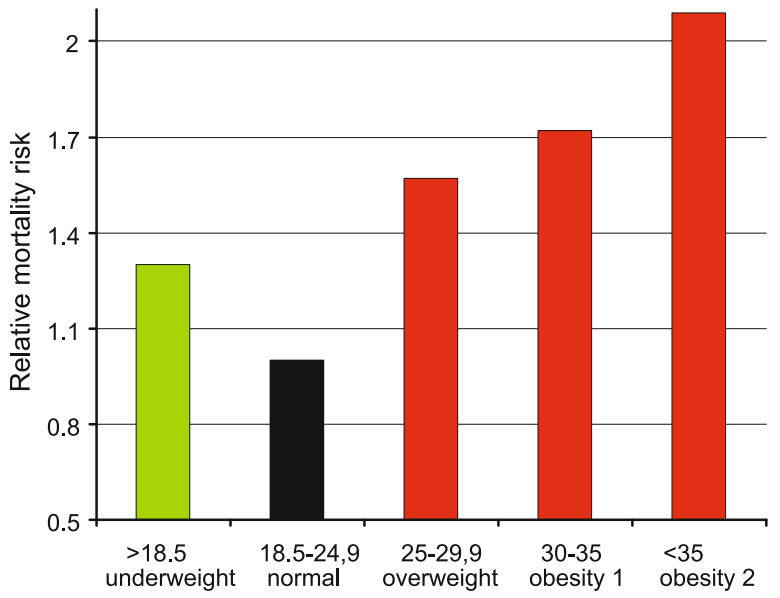

Fig. 5. Relative cardiovascular mortality risk associated with normal weight, overweight and obesity. According to Katzmarzyk et al (13).

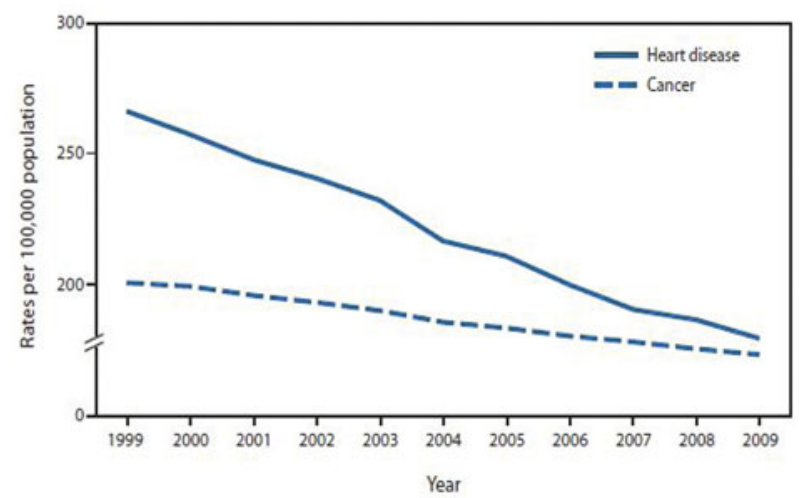

Fig. 6. Trends of standardized mortality caused by cardiovascular and cancer diseases in European Union. According to WHO (24).

India and Mexico. This report, based on data from 2.88 million individuals, linked to more than 270,000 deaths, detected no increase in mortality in overweight individuals (Fig. 3). An extensive dispute followed in attempts to clarify these unexpected findings.

It appears that much depends on the marker that defines overweight and obesity, whether it is a BMI or anthropometric measurements of WHR and WTR. BMI may be controversial because it is deemed to be less sensitive than the WHR. WHO and National Heart Lung Blood Institute classification of normal weight as a BMI 18.5 to $25 \mathrm{~kg} / \mathrm{m}^{2}$ obscures the fact that people with a BMI between 18.5 and $22 \mathrm{~kg} / \mathrm{m}^{2}$ have been found to have higher mortality than those with a BMI of 22-25 kg/m². Grouping these together raises the mortality rate for the normal-weight group, which could explain why their observed mortality is similar to those with grade 1obesity.

Doubt was raised (12) whether the current classification of individuals as "overweight" is appropriate, since there is little evidence of increased risk of mortality in this group. The Canadian report from 2012 (13) attempted to clarify controversies regarding BMI and mortality. Hazard ratios across successive BMI categories for all-cause mortality were 1.25 for low weight, 1.00 (reference-normal weight), 1.06 (overweight), 1.27 (obesity grade I)

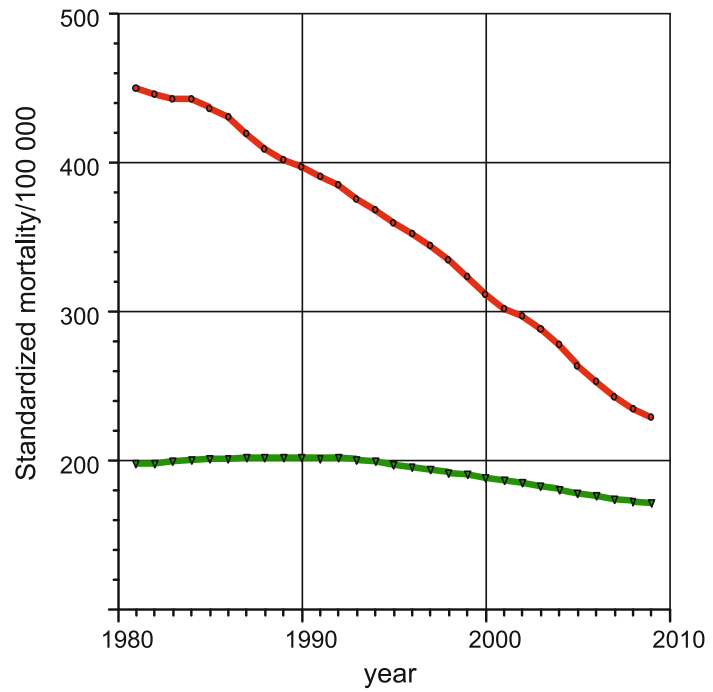

Fig. 7. Significant decrease of heart disease and cancer mortality in the whole US population. According to US National Vital Statistics System (25).

and 1.65 (obesity grade II). The difference between the normal weight and overweight was small and not significant.

Uncertainty regarding the most appropriate means, by which to express body weight, remains (14). Questions on BMI as an indicator of mortality generated numerous attempts at possible explanation that would support use of BMI: mortality in the obese may reflect a bias that heavier patients may be more aware of need for medical prevention and care, with better likelihood of receiving appropriate risk-factor treatment. In patients with chronic disease, BMI representing overweight may indicate a stable disease, linked with lower mortality. Even more speculative is the suggestion that in the absence of disease, moderate excess of adipose tissue may provide energy reserve for eventual catabolic illness.

While BMI has been used most widely, in recent years measures of central obesity like the waist circumference, but even more the WHR have been reported to more accurately indicate the distribution of body fat. Compared to BMI, these have been proposed to be more closely associated with morbidity and mortality (15). Selection of an appropriate indicator of adiposity makes mortality trends more realistic. A large cohort of men and women confirmed that WHR was positively associated with mortality in middle- aged adults. On the other hand, BMI and waist circumference exhibited U- or J-shaped association with mortality. These results clarify the seemingly unexplainable enigma of low mortality in overweight individuals identified on the basis of BMI (16).

Another report (17) analyzed data from a longitudinal study of high-functioning men and women, ages 70-79 years at baseline. They examined 12-year, all-cause mortality risk related to BMI, waist circumference and WHR. There was no association between all-cause mortality and BMI or waist circumference. However, the all-cause mortality increased with WHR. Additional reports (18, 19) indicated that the association between all-cause mortality and WHR is typical not only in the elderly but also in the middle age 
where the WHR, independently of BMI, strongly and positively predicted mortality, among both white and black adults. Such prognostic information appears to be further reaching beyond that provided by BMI alone.

\section{All-cause and cardiovascular mortality in overweight and obesity}

Another paradox observed while using BMI in relation to mortality, is the difference between all-cause mortality and cardiovascular (CVD) mortality (Fig. 4) (20). Risk of relative allcause mortality decreases from a higher value in subjects with abnormally low BMI toward low mortality both at normal BMI and at overweight BMI, while the relative CVD risk goes up both in overweight and obesity groups (20). According to other authors (13), the risk of CVD mortality increases even at overweight BMI and then grows rapidly in obesity (Fig. 5). Overweight determined by BMI is then not without risk: CVD mortality is increased and similar findings were confirmed in a large cohort also by others. WHR was confirmed to be significantly associated with risk of incident CVD events. For the British men, indices of body adiposity involving WHR had a stronger linear association with the odds of CVD than BMI (21).

A pressing dilemma persists: the epidemic of overweight and obesity appears to be disassociated with CVD mortality. CVD mortality in the USA declines while obesity continues to be on the rise. BMI is only one isolated measurement of how fit one is. Many other aspects of lifestyle such as exercise, stress relief, decrease in smoking, reduced intake of high calorie food and less exposure to toxic agents may have influenced pathogenesis of CVD more, than excess body weight of the population. In the past two decades, intensive preventive measures (control of hypertension, of blood lipid disorders) have been implemented.

Obesity pandemic has engulfed not only the affluent countries but also the developing world. Overweight and obesity are now so ubiquitous that they are quickly becoming an acceptable social norm while the risk for global health is reaching enormous proportions. It is important to deal with misleadingly beneficial conclusions based on various statistical trends that indicate potential absence of health risk in individuals who are overweight but not yet obese. Full impact of obesity on population health will become obvious in the near future, when present-day obese children and adolescents attain adult age (22). Adipocytes adjust to excess influx of fat in infancy and childhood, then present difficult-to manage obesity in adulthood. Use of a more sensitive indicator than the BMI for body fat distribution indicates that even overweight preceeding obesity has to be included as a potential health hazard. Overweight frequently represents a continuum before onset of obesity. Overweight with obesity had a potential impact on fatal and non-fatal complications of CVD (23).

\section{Conclusion}

While the prevalence of adiposity is dramatically increasing, LE is increasing as well. A parallel increase of adiposity and LE is not a paradox. The cardiovascular mortality and LE in the European Union and USA has improved. Important factor in LE is the infant mortality, which has been prominently decreasing in most of the economically advanced countries. In the past 2-3 decades, intensive preventive measures (control of hypertension, of cholesterol and triglyceride levels, better style of life, e.g. a decrease of smoking and increase of physical activity, etc) have been implemented both in people with normal weight and especially in patients with overweight or obesity, who are more aware of the need for medical prevention and care with a higher likelihood of receiving appropriate risk-factor treatment.

Heavy obesity shortens the LE. On the other side, improvement of health in individuals with normal weight, overweight and mild obesity (about 80-90\% of the population in economically developed countries) increases their LE and modifies the adverse outcome in the minority of heavily obese.

It is then conceivable that the general LE of the population as a whole is still improving. In the European Union and the United States there has been a prominent decline in CVD mortality (24, 25) and also a modest decline in cancer mortality (Figs 6 and 7). These favourable developments in mortality should not evoke undue optimism. There has been a lasting trend of increase in morbid obesity (Fig. 1). If the shift to higher grades of obesity continues despite attempts at effective preventive intervention, the steady rise in LE in the modern era may soon come to the end.

\section{References}

1. Ginter E, Simko V. Adult obesity at the beginning of the 21st century: epidemiology, pathophysiology and health risk. Bratisl Lek Listy 2008; 109 (5): 224-230.

2. Misra A, Singhal N, Khurana L. Obesity, the metabolic syndrome, and type 2 diabetes in developing countries: role of dietary fat and oils. $\mathrm{J}$ Am Coll Nutr 2010; 29 (3 Suppl: 289S-301S.

3. Wang Y, Beydoun MA. The obesity epidemic in the United States gender, age, socioeconomic, racial/ethnic, and geographic charcteristics: a systemic review and meta- regression analysis. Epidemiol Rev 2007; 29: 6-28.

4. Wang Y, Beydoun MA, Liang L et al. Will all Americans become overweight or obese? Estimating the progression and cost of the US obesity epidemic. Obesity (Silver Spring). 2008; 16: 2323-2330.

5. Tsai AG, Williamson DF, Flick HA. Direct medical cost of overweight and obesity in the USA: a quantitative systematic review. Obes Rev 2011; 12: 50-61.

6. Arterburn DE, Maciejewski ML, Tsevat J. Impact of morbid obesity on medical expenditures in adults. Int J Obes (Lond) 2005; 29 : 334-339.

\section{National Center for Health Statistics Health E-Stats, 2008, USA.}

8. U.S. Census Bureau, Statistical Abstract of the United States: 2012

9. Ginter E, Simko V, Dolinska S. Paradoxes in medicine: an access to new knowledge? Bratisl Lek Listy 2009; 110 (2): 112-115.

10. Berrington de Gonzalez A, Hartge P, Cerhan JR et al. Body-Mass Index and Mortality among 1.46 Million White Adults. N Engl J Med 2010; 363 (23): 2211-2219. 
11. Flegal KM, Kit BK, Orpana H, Graubard BI. Association of allcause mortality with overweight and obesity using standard body mass index categories: a systematic review and meta-analysis. JAMA 2013; 309 (1): 71-82.

12. McGee DL. Body mass index and mortality: a meta-analysis based on person-level data from twenty-six observational studies. Ann Epidemiol 2005; 15 (2): 87-97.

13. Katzmarzyk PT, Reeder BA, Elliott $S$ et al. Body mass index and risk of cardiovascular disease, cancer and all-cause mortality. Can J Public Health 2012; 103 (2): 147-151.

14. Huxley R, Mendis S, Zheleznyak E et al. Body mass index, waist circumference and waist: hip ratio as predictors of cardiovascular risk-a review of the literature. Eur J Clin Nutr 2010; 64: 16-22.

15. Seidell JC: Waist circumference and waist/hip ratio in relation to all cause mortality, cancer and sleep apnea. Eur J Clin Nutr 2010; 64: 35-41.

16. Reis JP, Araneta MR, Wingard DL et al. Overall obesity and abdominal adiposity as predictors of mortality in U.S.. white and black adults. Ann Epidemiol 2009; 19: 134-142.

17. Srikanthan P, Seeman TE, Karlamangla AS. Waist-Hip-Ratio as a predictor of all-cause mortality in high-functioning older adults. Ann Epidemiol 2009; 19: 724-731.

18. Kahn HS,Bullard KM,Barker LE et al Differences between adiposity indicators for predicting all-cause mortality in a representative sample of United States non-elderly adults. PLoS One 2012; 11: e50428.
19. de Koning L, Merchant AT, Pogue J et al. Waist circumference and waist-to-hip ratio as predictors of cardiovascular events: meta- regression analysis of prospective studies. Eur Heart J 2007; 28: 850-856.

20. Knecht S, Eliger T, Levine JA. Obesity in neurobiology. Progress Neurobiol 2008; 84: 85-102.

21. Cox BD, Whichelow MJ, Prevost AT. The development of cardiovascular disease in relation to anthropometric indices and hypertension in British adults. Int J Obes Relat Metab Disord 1998; 22: 966-973.

22. Garnett SP, Baura LA, Srinivasem $S$ et al. Body mass index and waist circumference in midchildhood and adverse cardiovascular disease risk clustering in adolescence. Am J Clin Nutr 2007; 86: 549-555.

23. van Dis I, Kromhout D, Geleijuse JM et al. Body mass index and waist circumference predict both 10-year nonfatal and fatal cardiovascular disease risk: study conducted in 20,000 Dutch women aged 20-65 years. Eur J Cardiovasc Prev Rehabil 2009; 16: 729-734.

24. European Health for All Database, WHO Regional Office for Europe, Copenhagen, updated January 2012.

25. National Vital Statistics System. Mortality public use data files for 1999-2007, and preliminary data for 2008 and 2009. From US Vital Statistics.

Received March 1, 2013. Accepted March 29, 2014. 